\title{
An analytical model for the microjet at the tip of a collapsing cavity
}

\author{
A. Crespo $\left({ }^{*}\right)$ and F. Manuel $\left({ }^{* *}\right)$ \\ E.T.S. Ingenieros Industriales, Universidad Politécnica de Madrid \\ José Gutiérrez Abascal 2, 28006 Madrid, Spain \\ Tel. : $34+1-5626200$. Fax : 34+1-5618618
}

J. Hernández $\left(^{*}\right)$

E.T.S. Ingenieros Industriales, Universidad de Murcia

Paseo Alfonso XIII 50, 30203 Cartagena, Spain

Tel. : 34+68-505912. Fax : 34+68-505310

\section{Introduction}

In turbulent flows, and in particular in turbulent boundary layers along the walls of turbomachine elements, there are multiple vortex filaments which may attract the gaseous products resulting from incipient cavitation, creating gaseous columns which may break into bubbles of a length of the order of magnitude of the radius of curvature of the filaments. In turbulent boundary layers there will be secondary vortex lines normal to the wall, and it is shown here that the resulting bubbles create during collapse very high velocities normal to the wall which may originate the cavitation damage. For very long bubbles, this damage is probably due to a microjet appearing at its tip ; the speed of the microjet is shown to be twice the speed of the tip (CRESPO et al. [1]), and very large compared to the typical collapse velocity of spherical bubbles. Microjets have also been observed in prolate spheroids in the theoretical calculations of PROSPERETTI

(*) Catedrático de Universidad.

(**) Profesor Titular de Universidad.
[2] and Shima and NAKajIma [3] ; however, they do not appear in the analysis of MILES [4]. A microjet also appears typically in situations such as in the bubble collapse in the presence of boundaries and in translating bubbles, as it is shown by BLAKE and GIBSON [5] and PLESSET and PROSPERETTI [6].

AVELLAN and KARIMI [7] made some experiments which consisted in creating a vortex subjected alternately to underpressure and overpressure by periodically opening and closing a valve. During the underpressure period a cavity appeared in the vortex nucleus, which subsequently collapsed against a wall normal to the vortex when the pressure was raised. In this wall a specimen was immersed and its erosion was observed. The side wall enclosing the vortex was transparent, and the diameter and length of the bubble were measured photographically as a function of time during the collapse ; these results are used for comparison with the results of the theory proposed in this paper. The collapse velocities and the damage to the specimen were substantially larger than those corresponding to the collapse of a spherical bubble, although a microjet has not been observed experimentally yet.

Un modèle analytique pour le microjet

à l'extrémité d'une bulle durant un collapse

L'écoulement est supposé irrotationel et la pression à l'interface constante. Une première approximation de la solution est obtenue en négligeant le terme de l'énergie cinétique dans l'équation de Bernoulli à l'interface. L'approximation est valable pour des temps courts, et commence à être fausse près du sommet, où deux microjets sont formés. L'évolution de la forme de la bulle et les caractéristiques des microjets sont estimées analytiquement et comparées avec les valeurs obtenues à partir de calculs numériques. 


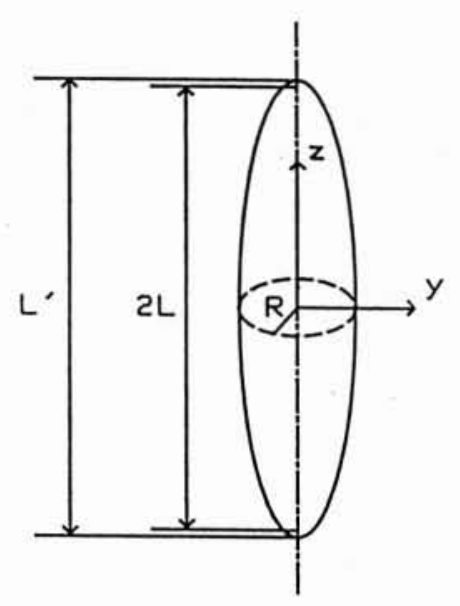

1. Schematic showing the sink-line model.

In this paper an analytical model is proposed to describe the collapse process of elongated bubbles appearing in the core of cavitating vortices. Viscous and turbulence effects are neglected, and the flow field is assumed to be irrotational. Although the vortex causes the initial elongated shape of the bubble, the radial variation of pressure due to the centrifugal force is neglected in the model presented here. If this radial pressure variation is comparable to the driving pressure causing the collapse, this approximation is no longer valid, and the resulting flow field may be quite different. CHAHINE [8] has performed numerical calculations showing that for large enough azimuthal velocities a constriction appears in the middle of the bubble, whereas in our problem there is a microjet at the tip. The effect of surface tension has not been considered either. This assumption may fail near the tip, where the radius of curvature is smallest. On the other hand, the nondimensional velocity in that region is largest, so that both effects cancel when combined in a Weber number, which turns out to be of an order of magnitude of $\Delta p L / \sigma$, with $\Delta p$ being the pressure difference between that at infinity and the saturated vapor pressure inside the bubble, and $\sigma$ is the surface tension : in many situations of interest this number is large.

The evolution of the bubble shape and a collapse time are firstly derived in Section 2 using a simplification which consists in neglecting the kinetic energy term in Bernoulli's equation. An expression is given for the collapse time that differs by a numerical factor from that obtained by MILES [4] for very elongated bubbles, using a variational method. In Section 3 a better approximation is introduced, retaining in Bernoulli's equation the kineticenergy term obtained in first approximation; the results allow to explain the formation of microjets at the tips of the bubble. In Section 4 the radius of the microjets is estimated from a global conservation of mechanical energy, and in Section 5 a comparison is made with the results of a numerical model based on the resolution of the irrotational flow equations using a singularity method.

\section{Description of the analytical model}

PROSPERETTI [2] suggests using distributions of sinks to describe the evolution of nonspherical bubbles during collapse. Based on this idea, it will be firstly assumed here that the flow field is due to a sink line of length $2 L$ and uniform intensity per unit length $Q(t)$ (fig. 1 ). In the following discussion, elliptical coordinates will be used defined by the transformation

$$
\begin{aligned}
& y=L \sinh \xi \sin \eta, \\
& z=L \cosh \xi \cos \eta .
\end{aligned}
$$

In this system of coordinates, surfaces of constant $\xi$ are confocal ellipsoids. The velocity potential is given by

$$
\phi=\frac{Q(t)}{4 \pi} \ln \left(\frac{\cosh \xi+1}{\cosh \xi-1}\right),
$$

so that surfaces of constant potential are ellipsoids of revolution whose foci are at the ends of the sink line (MILNE-ThOMSON, [9]).

The velocity, $\vec{v}=\nabla \phi$, is normal to the ellipsoids, and is given by

$$
v=-\frac{Q(t)}{2 \pi L} \frac{1}{\left(\cosh ^{2} \xi-\cos ^{2} \eta\right)^{1 / 2} \sinh \xi} .
$$

It may be pointed out that ellipsoidal bubbles with a velocity field at the surface given by equation (4) have a self-preserving shape (LAMB [10]). During the collapse, the bubble has to be a fluid surface (kinematic condition). If ir is considered that $L$ is a function of time $L(t)$ and that the potential surface moves with constant $\xi$, it turns out that the ellipsoid

$$
F(y, z, L(t))=\frac{y^{2}}{\sinh ^{2} \xi}+\frac{z^{2}}{\cosh ^{2} \xi}-L^{2}=0
$$

is a fluid surface if the following equation is satisfied :

$$
\vec{v} \cdot \nabla F+\frac{\partial F}{\partial L} \frac{\mathrm{d} L}{\mathrm{~d} t}=0 .
$$

Using equations (4) and (5), from equation (6) an expression for $L(t)$ is obtained,

$$
\frac{\mathrm{d} L^{2}}{\mathrm{~d} t}=-\frac{Q(t)}{\pi} \frac{1}{\sinh ^{2} \xi \cos \xi},
$$

where $\cos \eta$ has disappeared, as anticipated, and $Q(t)$ has to be calculated. The pressure on the bubble has to be constant (mechanical condition) and equal to the vapor pressure. Assuming that the fluid is at rest at infinity, where there is a constant pressure $p_{\infty}$, Bernoulli's equation at the bubble surface can be written as

$$
\frac{\mathrm{d} \phi}{\mathrm{d} t}=\frac{\partial \phi}{\partial t}+\vec{v} \cdot \nabla \phi=\frac{\partial \phi}{\partial t}+v^{2}=\frac{p_{\infty}-p}{\rho}+\frac{v^{2}}{2},
$$

where $p$ is the vapor pressure, and the total time derivative of $\phi$ at the bubble surface has been used instead of the partial derivative. If it is assumed that the bubble maintains its initial shape during collapse, to 
satisfy the mechanical condition it is necessary that surfaces of constant potential are surfaces of constant pressure ; this may be approximately true in the initial stages of the collapse process, when it can be assumed that $v \approx 0$, but not later. Neglecting the term $v^{2} / 2$ and integrating equation (8) yields

$$
\phi=\frac{\Delta p}{\rho} t .
$$

From equation (3) it follows that

$$
Q(t)=4 \pi \frac{\Delta p}{\rho} t\left[\ln \left(\frac{\cosh \xi+1}{\cosh \xi-1}\right)\right]^{-1},
$$

and, by substituting this equation into equation (7), it is found that

$$
L=L_{0} \sqrt{1-\tau^{2}}
$$

where

$$
\tau=t / t_{c},
$$

and

$$
\begin{aligned}
& t_{c}= \\
& =\left\{\frac{L_{0}^{2} \ln [(\cosh \xi+1) /(\cosh \xi-1)] \sinh ^{2} \xi \cosh ^{2} \xi}{2 \Delta p / \rho}\right\}^{1 / 2}
\end{aligned}
$$

$t_{c}$ is the collapse time and $L_{0}$ is the initial value of $L$. Equation (11) is slightly different from that obtained by CRESPO et al. [1], in which $L$ tends to a finite value for $\tau=1$. In figure 2 a comparison is made of the evolution of the bubble length, $L^{\prime}=L \cosh \xi$, and diameter, $2 R=2 L \sinh \xi$, obtained from equation (11) (the bubble shape is preserved) with the experimental results by AVELLAN and KARIMI [7]. The agreement between theory and experiments is good and better than that previously obtained by CRESPO et al. [1]. The presence of a wall normal to the bubble is taken into account by putting the wall at the middle of the bubble, so that the real bubble length will be half of that in figure 2. This relative position of the bubble with respect to the wall corresponds to the configuration of AVELLAN and KARIMI [7] experiments and to bubbles associated to the secondary vorticity of turbulent boundary layers.

For elongated bubbles $(\sinh \xi=\varepsilon \ll 1)$, the collapse time

$$
t_{c}=\frac{L_{0} \varepsilon[\ln (2 / \varepsilon)]^{1 / 2}}{(\Delta p / \rho)^{1 / 2}},
$$

and the axial velocity of collapse is of an order of magnitude

$$
\frac{L}{t_{c}} \sim \frac{(\Delta p / \rho)^{1 / 2}}{\varepsilon[\ln (2 / \varepsilon)]^{1 / 2}},
$$

so that it is a decreasing function of $\varepsilon$; the collapse will be intense if the bubble is very elongated. Expression (14) differs by a factor of 0.89 from the result of Miles [4].
In the limit $\xi \rightarrow \infty$, the ellipsoid becomes a sphere of radius $R_{s}=L \sinh \xi$, and the collapse time is given by

$$
t_{c}=R_{s}\left(\frac{\rho}{\Delta p}\right)^{1 / 2},
$$

which turns out to be a good approximation to the classical Rayleigh expression,

$$
t_{c}=0.915 R_{s}\left(\frac{\rho}{\Delta p}\right)^{1 / 2} .
$$

Although equation (11) is only valid at the initial stages of the collapse process, the good agreement between equations (16) and (17) could be due to the fact that the implicit assumption in equation (9) perhaps holds during a relatively long period of time in the collapse process. As it will be shown later, equation (11) is expected to be valid for the whole collapse process if $\xi \ll 1$.

\section{A further approximation}

If equation (4) is used to calculate the kinetic energy, equation (8) can be written as

$$
\frac{\mathrm{d} \phi}{\mathrm{d} t}=\frac{\Delta p}{\rho}+\frac{Q^{2}}{8 \pi^{2} L^{2}} \frac{1}{\left(\cosh ^{2} \xi-\cos ^{2} \eta\right) \sinh ^{2} \xi} .
$$

If $r_{1}$ and $r_{2}$ are the distances to the foci from a point in the interface of coordinates $(\xi, \eta)$, it can be written that

$$
\frac{\mathrm{d} \phi}{\mathrm{d} t}=\frac{\Delta p}{\rho}+\frac{Q^{2}}{16 \pi^{2} L \sinh ^{2} \xi \cosh \xi}\left(\frac{1}{r_{1}}+\frac{1}{r_{2}}\right) .
$$

Equation (10) may be substituted into equation (19) (terms of order $t^{3}$ are therefore neglected) giving, after integration,

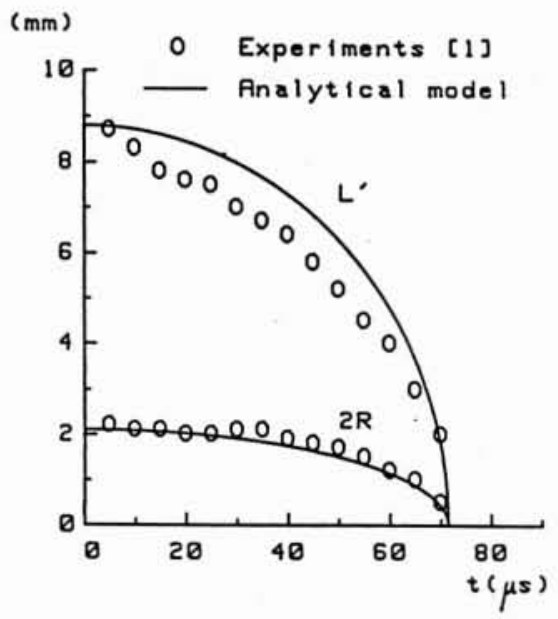

2. Bubble length and diameter as a function of time. Comparison with experiments [7]. Slenderness ratio $\varepsilon=0.12 ;$ driving pressure : $\Delta p \approx 6$ bar. 


\section{A. CRESPO et al.}

$\phi=\frac{\Delta p}{\rho} t+$

$+\frac{(\Delta p / \rho)^{2} t^{3}}{3 L \sinh ^{2} \xi \cosh \xi}\left[\ln \left(\frac{\cosh \xi+1}{\cosh \xi-1}\right)\right]^{-2}\left(\frac{1}{r_{1}}+\frac{1}{r_{2}}\right)$,

which is the sum of the velocity potential due to a sink line of uniform intensity given by equation (10), located between the foci of the ellipsoid defined by equation (5), and that due to two sinks located at the foci of intensity given by

$$
Q_{1}=\frac{4 \pi(\Delta p / \rho)^{2} t^{3}}{3 L \sinh ^{2} \xi \cosh \xi}\left[\ln \left(\frac{\cosh \xi+1}{\cosh \xi-1}\right)\right]^{-2} .
$$

If $\xi \ll 1$ (i.e., for elongated bubbles), the effect of the two sinks would be local and negligible over most of the bubble surface; moreover, the velocities induced at points located far enough from the sinks would be not only small but also parallel to the interface. According to this result, the effect of the kinetic energy term in Bernoulli's equation will be a local one associated to singularities near the tips, so that the sink-line model will be valid over most of the bubble surface, except in a small region near the tip, and most of the bubble surface would evolve as a self-preserved ellipsoid whose halfdistance between foci is given by equation (11). However, the integration made to obtain equation (20) may be shown to be uniformly valid only during the first stages of the collapse process, $\tau \ll \varepsilon \ll 1$; for longer times and in regions close to the tip, it is not possible to consider the distances to the foci as constants in the integration, and a microjet appears, as described in the quasi-steady model proposed by CRESPO et al. [1]. This model for the microjet is based on a simple model for shaped charges proposed by BATCHELOR [11], and may be described as follows : near the tip, in a reference frame moving with the tip velocity, the flow is quasi-steady and the velocity at the interface is tangent to it and constant, equal to the tip velocity; then, in an absolute reference frame the microjet velocity is twice the tip velocity. It may be shown, using a singular perturbation method, that this quasi-steady approximation is valid for $\varepsilon \ll \tau \ll 1$ in a region where $\eta \ll \tau$, whereas the velocity field tangent to the interface due to the sinks given in equation (21) is valid for $\eta \gg \tau$; there is an analytical solution matching these two flow fields.

\section{Radius of the microjet}

In this section, the integral equation for conservation of mechanical energy is used to estimate the microjet radius in the limit $\sinh \xi=\varepsilon \rightarrow 0$.

The kinetic energy in the flow field may be expressed as

$$
K_{1}=\int_{V} \rho \frac{v^{2}}{2} \mathrm{~d} V=\frac{\rho}{2} \int_{S} \phi \frac{\partial \phi}{\partial n} \mathrm{~d} S
$$

where $S$ is the interface. For the flow without microjet, $\phi$ is given by equation (3), and

$$
\begin{aligned}
K_{1}=\rho \frac{Q^{2}}{4 \pi} \ln (2 / \varepsilon) 2 L & \\
= & \frac{2 \rho \pi \tau^{2} R_{0}^{5} \ln (2 / \varepsilon)}{\varepsilon t_{c}^{2}} \sqrt{1-\tau^{2}} .
\end{aligned}
$$

The decrease of potential energy during collapse, neglecting the volume of the microjet provided that $\varepsilon$ is small enough, may be written as

$$
\begin{aligned}
P_{1}=\Delta p \Delta V= & \frac{4}{3} \pi R_{0}^{3} \varepsilon^{-1}\left[1-\left(1-\tau^{2}\right)^{3 / 2}\right] \Delta p= \\
& =\frac{4}{3} \pi \rho R_{0}^{5} \frac{\ln (2 / \varepsilon)}{\varepsilon t_{c}^{2}}\left[1-\left(1-\tau^{2}\right)^{3 / 2}\right] .
\end{aligned}
$$

The difference between the energy potential decrease and the calculated kinetic energy without microjets is made equal to the kinetic energy of the two microjets :

$$
P_{1}-K_{1}=2 \times \frac{1}{2} \rho V_{M}\left(2 \frac{\mathrm{d} L}{\mathrm{~d} t}\right)^{2},
$$

where $V_{M}$ is the volume of each microjet, and $2 \mathrm{~d} L / \mathrm{d} t$ is the absolute microjet velocity, as shown in [1].

From the three previous equations it is obtained that $V_{M}=$

$$
{ }_{0}^{3} \varepsilon \ln (2 / \varepsilon) \frac{1-\tau^{2}}{\tau^{2}}\left[\frac{1}{3}-\sqrt{1-\tau^{2}}\left(\frac{1}{3}+\frac{\tau^{2}}{6}\right)\right],
$$

and in the limit $\tau \ll 1$,

$$
V_{M}=\frac{\pi}{8} R_{0}^{3} \varepsilon \ln (2 / \varepsilon) \tau^{2} .
$$

using again the microjet model to estimate its length,

$$
L_{M}=L_{0}-L=L_{0}\left(1-\sqrt{1-\tau^{2}}\right) \simeq L_{0} \frac{\tau^{2}}{2} .
$$

For $\tau \ll 1$ the microjet radius then becomes independent of $\tau$ :

$$
R_{M}=\frac{R_{0}}{2} \varepsilon\left[\ln \left(\frac{2}{\varepsilon}\right)\right]^{1 / 2}
$$

\section{Comparison with results of a numerical model}

To check the validity of the model proposed here, a numerical singularity method has been developed. The irrotational flow field is obtained by superimposing the flow fields of $N$ sink rings, located in the inside normal of $N$ points of the bubble surface, where the flow properties have to be calculated. For very elongated bubbles, this numerical procedure is very unstable, and diverges in a short time. Nevertheless, the calculations were carried out for sufficient time, and enough numerical data has been obtained to validate the analytical model. A more detailed description of the numerical model can be found in [1]. 
The results of the numerical model confirm the existence of a microjet at the tip of the bubble. Figure 3 shows the numerical predictions for the evolution during collapse of the shape of initially ellipsoidal bubbles of different slenderness ratios.

The microjet radius obtained from the numerical results may be compared with the values given by equation (29). It may be seen that for the cases of figure 3 the agreement is good, and better for the case of $\varepsilon=1 / 4$, as expected. Numerical results also show that the microjet radius is independent of $\tau$, what is in agreement with equation (29).

\section{Conclusions}

The proposed analytical model gives a description of the collapse process of elongated bubbles appearing in the core of cavitating vortices. For the first stages of the collapse process, a first approximation, consisting in neglecting the kinetic-energy term in Bernoulli's equation, has been introduced; it predicts a collapse in which the bubble maintains its initial ellipsoidal shape, while the collapse is due to a sink line of uniform intensity. In a second approximation the kinetic energy in Bernoulli's equation is made equal to that obtained in first approximation. The resulting velocity potential is found to be equal to the sum of that due to a sink line of uniform intensity, located between the foci of an ellipsoid, and that due to two sinks of certain intensity located at the foci. This solution is related with the formation of microjets at the tips of the bubble. It also serves to justify the assumption that, for very elongated bubbles, the effect of the kinetic energy term in Bernoulli's equation is a local one and that the collapse process over the whole interface, excluding a small region near the tips, is described by the sink-line model. An estimation of the radius of the microjets has been obtained from a global conservation of mechanical energy. The comparison of the estimated values with the results obtained from a numerical model based on the resolution of the irrotational flow equations gives a good agreement. The comparison with experiments of AVELLAN and KARIMI [7] is better than that obtained with the model previously presented in [1].

\section{Acknowledgements}

This work has been supported by Spanish grants « Acción Integrada Hispano-Francesa $» n^{\circ} 86 \mathrm{~B}$ and research project PB $89 / 0184$ of the DGICYT.

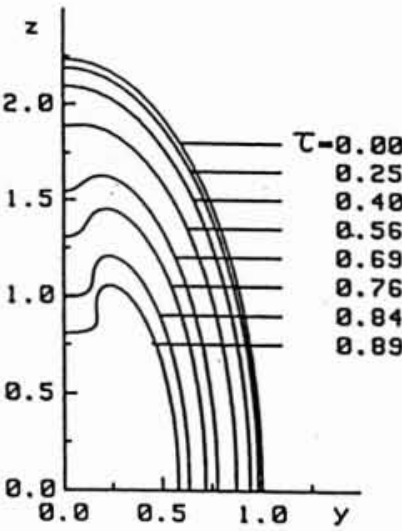

(a)

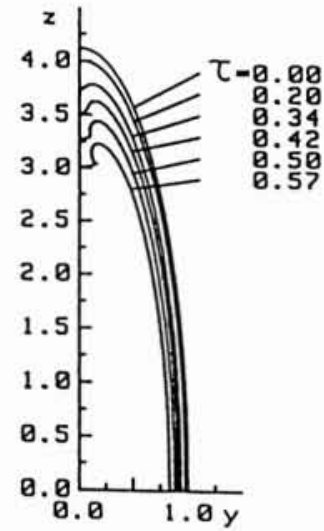

(b)
3. Numerical results for the evolution of a bubble during collapse. (a) $\varepsilon=1 / 2$; (b) $\varepsilon=1 / 4$.

\section{References}

[1] Crespo A., Castro F., Manuel F. and Hernández J. « Dynamics of an Elongated bubble During Collapse ", ASME Journal of Fluids Engineering 112, pp. 232-237, 1990.

[2] Prosperetti A. - «On the Dynamics of Nonspherical Bubbles ", Proc. of the First International Conference on Cavitation and Inhomogeneities in Underwater Acoustics, Göttingen, 1979.

[3] Shima A. and Nakajima K. - « The Collapse of a Nonhemispherical Bubble Attached to a Solid Wall ", Journal of Fluid Mechanics 80, pp. 369-391, 1977.

[4] Mnles J.W. - «The Collapse Time of a Closed Cavity », Journal of Fluid Mechanics 25, pp. 743-760, 1966.

[5] BlaKe J.R. and Gibson D.C. - « Cavitation Bubbles Near Boundaries ", Annual Review of Fluid Mechanics 19, pp. 99 125, 1987.

[6] Plesset M.S. and Prosperetti A. - « Bubble Dynamics and Cavitation ", Annual Review of Fluid Mechanics 9, pp. 145-185, 1977.

[7] Avellan F. and Karimi A. - «Dynamics of Vortex Cavitation Involved in the Erosion of Hydraulic Machines ", Proc. VII International Conference on Erosion by Liquid and Solid Impact, Cambridge, 1987.

[8] Chahine G.L. - «A numerical Model for Three-Dimensional Bubble Dynamics in Complex Flow Configurations ", 2nd ATTC Meeting, St. John's, Newfoundland, Canada, 1989.

[9] Minne-Thomson L.M. - Theoretical Hydrodynamics, Macmillan, Ex. 7, Chapter XV, 1960.

[10] LAMB H. - Hydrodynamics, 6th Ed. 1932, Dover, Chapter 5, 1945.

[11] BATChelor G.K. - An Introduction to Fluid Dynamics, Cambridge University Press, Section 6.3, 1967. 


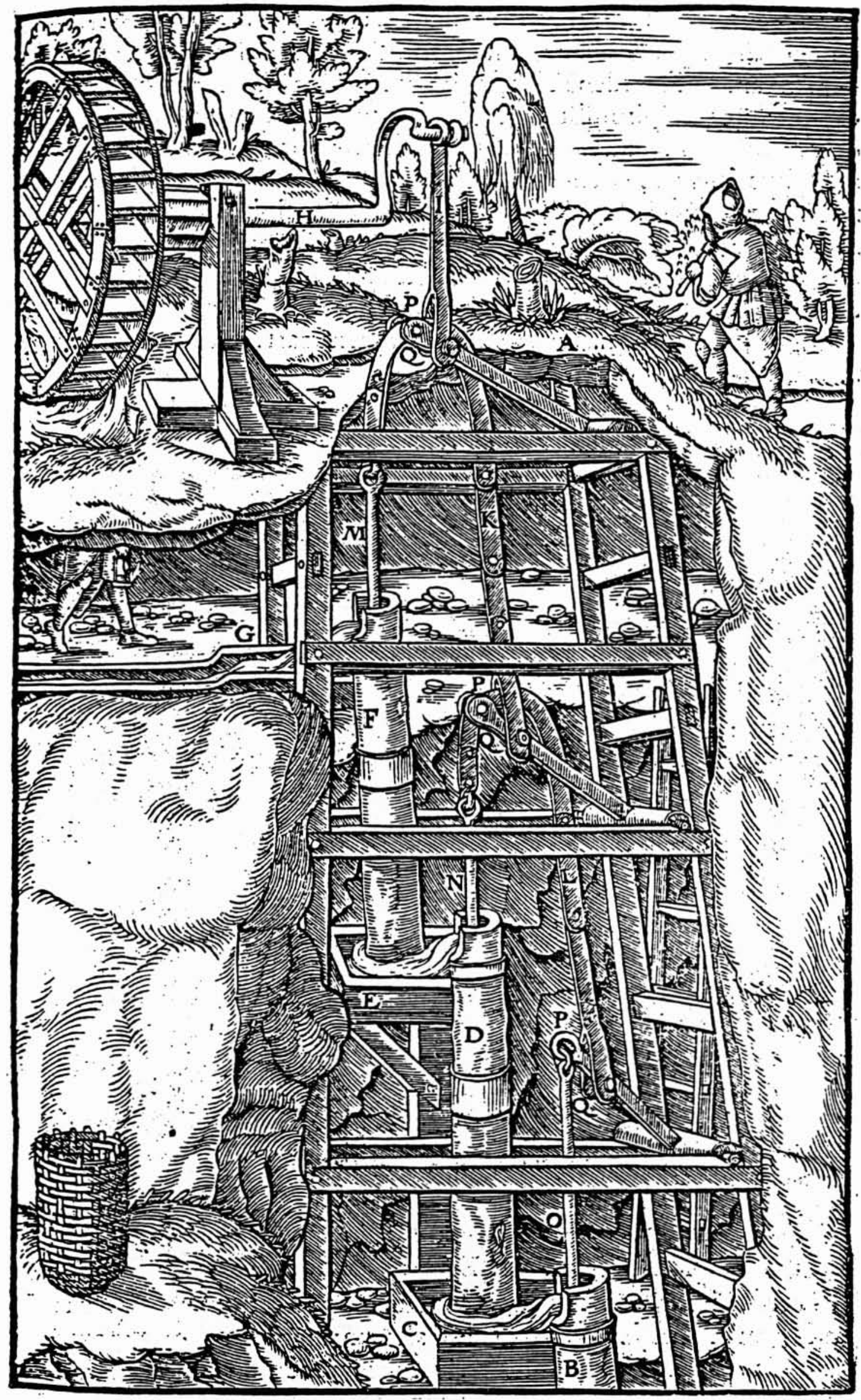

Agricola (G.) - De re metallica (1556) 